\title{
Archival Data Repository Services to Enable HPC and Cloud Workflows in a Federated Research e-Infrastructure
}

\author{
Sadaf R. Alam*, Javier Bartolome ${ }^{\dagger}$, Sanzio Bassini ${ }^{\ddagger}$, Michele Carpene ${ }^{\ddagger}$, Mirko Cestari ${ }^{\ddagger}$, Frederic Combeau ${ }^{\S}$, \\ Sergi Girona ${ }^{\dagger}$, Stefano Gorini*, Giuseppe Fiameni ${ }^{\ddagger}$, Björn Hagemeier ${ }^{\Uparrow}$, Thorsten Hater ${ }^{\Uparrow}$, Andreas Herten ", \\ Nikoleta Kiapidou $^{\dagger}$, Wouter Klijn $₫$, Dorian Krause ${ }^{\Uparrow}$, Jacques-Charles Lafoucriere ${ }^{\S}$, Cerlane Leong*,

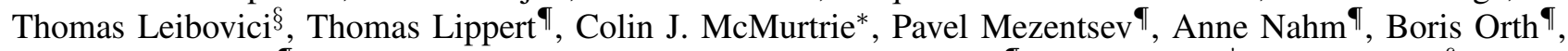 \\ Dirk Pleiter ${ }^{\uparrow}$, Thomas C. Schulthess*, Benedikt von St. Vieth ${ }^{\uparrow}$, Debora Testi ${ }^{\ddagger}$, Gilles Wiber ${ }^{\S}$ \\ ${ }^{*}$ CSCS, Swiss National Supercomputing Centre, 6900 Lugano, Switzerland \\ ${ }^{\dagger}$ Barcelona Supercomputing Center, 08034 Barcelona, Spain \\ ${ }^{\ddagger}$ CEA DAM, 91680 Bruyères-le-Châtel, France \\ $\S$ CINECA, 40033 Casalecchio di Reno, Italy \\ ๆ Jülich Supercomputing Centre (JSC), Forschungszentrum Jülich, 52425 Jülich, Germany
}

\section{Abstract}

Five European supercomputing centres, namely BSC (Spain), CEA (France), CINECA (Italy), CSCS (Switzerland), and JSC (Germany), agreed to align their high-end computing and storage services to facilitate the creation of the Fenix Research Infrastructure. In addition to the traditional extreme-scale computing and data services, Fenix provides a set of Cloud-type services as well as services needed for federation. In this paper, we describe the architecture of the Fenix infrastructure and how it can be used for representative workflows from the Human Brain Project (HBP). The concept of the Active Data Repository (ACD) is chosen to highlight demarcation between HPC and Cloud access models.

\section{BACKGROUND AND Motivation OF FeniX}

Many of the workflows using High Performance Computing (HPC) systems used to consist of simulations that consume and produce data typically confined to a single data centre. Data staging from external sources is periodic, with well-defined interfaces, unlike on-demand and streaming requirements of typical cloud workloads. An increasing number of research projects, however, extend beyond the HPC centre, in particular those processing data from large experimental facilities and other, unstructured data sources. These workflows are executed as a joint effort involving scientists from different sites and require different types of resources, including HPC resources.

One research domain that is implementing such workflows and that is working towards creating an IT-based research infrastructure supporting collaborative research is the neuroscience community organised in the HBP. One of the key aims of the European Human Brain Project is to build a research infrastructure (RI), recently branded as EBRAINS [3], to support research and development by the neuroscience communities. The Fenix Infrastructure provides services that will be used by EBRAINS, which is building-up a service portfolio for different types of brain research.

In the Fenix consortium there is a strong commitment for a use-cases driven co-design process for realising the Fenix architecture. Its features have been realised through a careful selection of services comprising consumable resources, underlying technologies enabling federation and services supporting governance and business processes. The focus has been operational sustainability of services, therefore, opensource and open-standard technologies have been adopted for implementation of the architecture.

\section{Fenix Compute and Data Services}

The Fenix research infrastructure comprises different compute and data services in combination with a thin layer of federation services. The latter includes federated identity and access services, as well as resource management and accounting services. The compute and data services fall into two broad categories, those that are operated in a restricted HPC environment and those that are operated in a more open, Cloud-like environment.

The Scalable Compute Services (SCC) abstracts large-scale computing resources contributed by the various partnering sites; usually massively parallel HPC systems with access

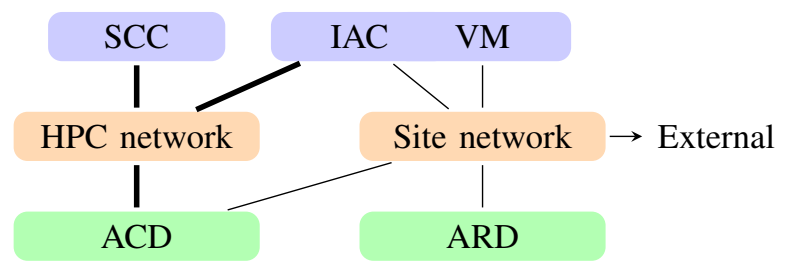

Fig. 1. High-level base architecture for instantiation by partnering sites, where the services will be provided by complementing and extending the local infrastructure. 
to accelerator hardware. The primary domain of these resources are highly parallel computations or high-throughput data analysis tasks. While SCC resources continue to be provided in a batch-type manner, an increasing need for supporting interactive work has been identified. Fenix' Interactive Compute Services (IAC) allow for ad-hoc access to single compute servers. Typical use cases are interactive analyses, visualisations, and steering of simulations running on SCC. Virtual Machine (VM) services offer access to on-demand Linux hosts via the OpenStack platform, which can also be used for long running services. Provisioning offers networking infrastructure including outside access through the internet and basic security via firewall rules.

Facilitating shared access to federated data stores as well as high-performance access to data stores from large-scale HPC systems is a challenge due to incompatible requirements, ranging from performance requirements to idenity and access models to functional abstractions. Fenix addresses this challenge by introducing different classes of data repositories, namely Archival Data Repository (ARD) and ACD, plus a Data Mover service for copying or moving data between these two types of data stores. The Data Mover is a service that is currently under development. One of the key features is integration of HPC resources management and scheduling system in order to facilitate orchestration of workflows across the data stores. $A R D$ is a federated data store optimised for capacity, reliability, and availability, used for long-term storage of large data sets that cannot be easily recreated. Data sets can be stored for the duration of the project to which the corresponding resources have been allocated. In practice, this means that data can be kept for one or few years. To meet modern requirements for long-term preservation of scientific data, a (small) fraction of the available capacity can be used for storing data for up to ten years on specific requests. These repositories are implemented on-top of the OpenStack Swift object store protocol. This ensures coherent access control within the federated infrastructure, through an open protocol, across all Fenix sites. An important feature is that ARD is accessible from services deployed in both, HPC and Cloud environments (in addition to a public internet endpoint). Typically, access requires authentication, but Swift also allows making containers openly accessible.

The moniker $A C D$ describes storage resource close to computational resources that can be used for storing working sets to be consumed by jobs in the SCC. These are typically implemented using parallel file systems. Access to ACD is considered to be local to a single partner site.

\section{Approach to Federation of Services}

The Fenix Research Infrastructure delivers federated compute and data services by aggregating capacity from multiple resource providers and enabling access from existing community platforms, such as the collaboratory of the HBP. In order to achieve these goals, the federation relies on a common Authentication and Authorisation Infrastructure (AAI), a trustworthy environment where users and resources can be managed at a federation level. The Fenix AAI provides central access to infrastructure services, e.g. storage and virtualisation, while platform services or community platforms will retain their own authentication and authorisation mechanisms.

As the federation of distributed resources through establishing a common AAI has been largely discussed within other research infrastructures in Europe, our strategy is based on the blueprint architecture of the Authentication and Authorisation for Research and Collaboration (AARC) project [13]. The AARC project is an European initiative providing guidelines, solutions and best practices to address the need for federated access to digital infrastructures. Thus, according to the design principles presented above, and the outcomes of the AARC project, our approach foresees the deployment of two services covering the following high-level functions respectively:

- Fenix Central Proxy IdP

- User identification and authentication

- Federation of multiple IdPs by proxying authentication requests

- Validation of user profiles

- Policy registry and management of principles of engagement

- Managing general Fenix Usage Agreement

- Fenix User and Resource Management Services (FURMS)

- Group/budgets membership management

- Authorisation attributes provider

- SSH public keys management

- Managing the site specific Usage Agreements

- Reporting and metering

The reason behind the creation of these two services is twofold: keep the Central Proxy IdP as lean as possible in order to provide high operational performance; improve infrastructure security by decoupling highly critical functions from less critical ones. As a result, operation of the Central Proxy IdP, for Fenix AAI, has been delegated to an external, highly accredited entity, namely GÉANT [12].

The Fenix Central Proxy IdP is responsible to proxy authentication requests among hosting site's IdPs. At the same time each site is deputed to maintain its own IdP, plus as many Service Providers (SPs) as the number of offered services. Through the Central Proxy IdP, a user already registered on a site or an external IdP (e.g. eduGAIN, HBP) will be able to access services and resources provided by other Fenix sites without registering twice and using its home IdP to authenticate. The Central Proxy IdP is also responsible to control the validity of a user profile, including the acceptance of the Fenix access policy, and to provide a set of APIs to permit hosting sites, or other services, to retrieve information about user profile basic attributes.

The Fenix Central IdP does not enforce access rights but simply forwards authenticated users' attributes to the hosting site which, applying local policies, decides if a user can access services or not. From the technical point of view, the Central Proxy IdP is implemented using the SATOSA 


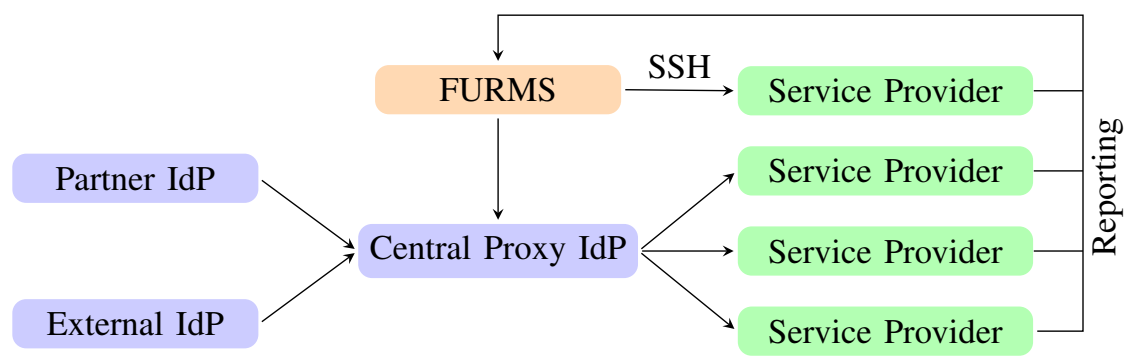

Fig. 2. High-level Fenix AAI concept. Users authenticate against a local IdP, either at a Fenix site or an external partner. These credentials are forwarded to the Central Proxy IdP who validates the requests and provides a new set of credentials that can be used across all service providers. The user's role, project memberships, etc is managed through the attribute provider FURMS. These attributes are used to implement accounting, access control, and similar policies. While not explicitly stated, service providers can use different protocols for authentication, where FURMS has the special role of managing SSH keys used for direct access. This is a common requirement in HPC environments.

[21] technology. Essentially, Fenix AAI is an instantiation of GÉANT eduTEAMS Dedicated service. This service offers communities requiring full control of their AAI where GÉANT can host and operate their own, dedicated AAI Service powered by the eduTEAMS technology [5]. Fenix community therefore relies on the operational capabilities and expertise of GÉANT, while remain in full control of the policies, configuration and branding of their AAI. Throughout the implementation process open standards and tools have been adopted to ensure scalability and extensibility of the federated AAI solution across multiple domains and institutions. For instance, eduTEAMS enables members of the research and education community to create and manage virtual teams and securely access and share common resources and services using federated identities from eduGAIN and trusted Identity Providers. A standard set of attributes and schemes are used to ensure interoperability across federations, such as eduPerson [19].

The proxy will be also configured to release Level of Assurance (LoA) information for each federated IdP, as well as the IdP the user is authenticating from. At the initial implementation stage, LoAs are defined as following:

- HIGH (Fenix hosting site IdP. Currently, this includes BSC, CEA, CINECA, CSCS and JSC)

- MEDIUM (home organization IdP, e.g. Human Brain, PRACE)

- LOW (unreliable identity verification)

If needed, the Fenix Central Proxy IdP may also retrieve attributes from external Attribute Authorities to enrich user profiles by attributes relevant for decisions by the service providers. The current implementation supports widely used protocols such as SAML and OIDC. Table I lists the attributes released to a service.

Conversely, FURMS acts essentially as a Service Provider (SP) of the Central Proxy IdP and will be responsible for managing and issuing authorization records, such as information about user budgets on hosting sites. In order to manage allocation and accounting of multiple resources across the federated sites, resources are made available by sites as Fenix credits. Fenix credits represent units of value for consuming specific resources at site. They comprise four attributes:

\begin{tabular}{|l|l|}
\hline displayname & urn:oid:2.16.840.1.113730.3.1.24 \\
\hline mail & urn:oid:0.9.2342.19200300.100.1.3 \\
\hline subject-id & urn:oasis:names:tc:SAML:attribute:subject-id \\
\hline eduPersonUniqueID & urn:oid:1.3.6.1.4.1.5923.1.1.1.13 \\
\hline eduPersonScopedAffiliation & urn:oid:1.3.6.1.4.1.5923.1.1.1.9 \\
\hline eduPersonAssurance & urn:oid:1.3.6.1.4.1.5923.1.1.1.11 \\
\hline eduPersonEntitlement & urn:oid:1.3.6.1.4.1.5923.1.1.1.7 \\
\hline
\end{tabular}

ATtributes RelEASED to SERVICES

1) Type of resource

2) Amount of this resource

3) Fenix resource provider (Site, at which it can be consumed)

4) Validity period

Periodically, information on available and planned resources are communicated via the Fenix website [9]. Within a type of service, for instance, the SCC, resources are currently available from two Fenix sites. The resources are rather diverse such that users and communities can decide based on the suitability of their workloads and usage scenarios. At the same time, we aim for a high level of uniformity for at least some services. This reduces complexity from a user perspective and can facilitate realising resilience and high availability within the distributed infrastructure by means of replication. Uniformity can be achieved in different ways. Let us consider the case of VM services. Here we use a standard technology, namely OpenStack. While the underlying hardware is different at the different sites, the offered range of VM models has been standardised within Fenix [10]. This approach allows platform developers to migrate and load balance across multiple OpenStack instances.

Multiple access mechanisms are supported fore requesting access to these resources that involve a range of evaluation processes for technical feasibility and scientific impact.

\section{A. Usage Example: Federated Long-term Storage}

To give an example for a federated workflow, we describe a typical interaction with the federated storage architecture offered by Fenix, the ARD. As mentioned earlier, ARD is a federated data store instantiated using the OpenStack Swift object store protocol. This decision has been motivated by a 


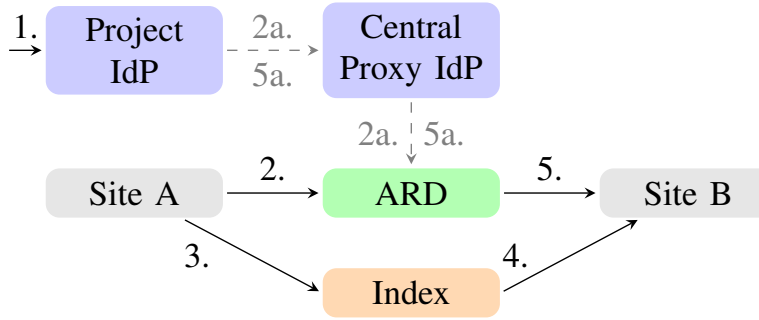

Fig. 3. Federated storage workflow, see text for the steps.

few factors. First, the protocol must support the underlying identity and access management (IAM) federation i.e. Fenix AAI. Second, the protocol must of open standard and open technology. Lastley, the technology must support features and abstractions necessary for incorporating cloud and HPC workflows such as support for anonymous accesses, georeplication, handling of small-to-large objects to name a few. In short, the solution should support the separation of concerns between platforms developers such as EBRAINS and Fenix infrastructure services providers. On one hand, the platform developers have freedom to develop their frameworks and tools knowing that their solutions are not limited to the Fenix resources and can be extended to other IT research infrastructure. On the other hand, the Fenix sites have autonomy to extend and manage life cycle of their infrastructure services with open, standard abstractions, APIs and protocols. Consequently, Fenix community can grow beyond the initial sites and can support a set of generic services that can be explited by a range of community platforms.

Swift is OpenStack project for the object store [16]. It offers several features needed for the Fenix ARD service. This includes a simple API abstraction, compliance with the AAI protocols, high availability and extensibility and above all support from an open community such as OpenStack. A full OpenStack installation is not needed because the API can be supported on a file system based solution namely Swiftonfile [17]. In fact, the initial installation at CSCS, a Fenix site, exploits IBM SpectrumScale for object storage to provide a Swift-compliant object store. The characteristics features of the Swift API include discoverability, flexible management of containers and objects, role based access controls, fine grain access controls, and custom management of large objects. Users and platform developers have flexibility for managing resources both from the OpenStack Horizon dashboard as well as command line interface (CLI) for managing workflows for data transfer and location services.

An object store offers key concepts and functionalities for management of data and storage. Key concepts include container and objects. A container is essentially a namespace for objects. An object can store data contents such as documents or images. Key functionalities for an object service include listing of objects and containers for search and discoverability, and management of objects and containers for access control, uploading, downloading and deleting items, etc. Additional control features are available for metadata that permits a user to gather statistics and monitor access to containers and objects.

As the infrastructure is still in construction, this workflow can not yet be realised fully. First, we describe the user's intention in this transaction, i.e. a data item is produced at a Fenix partner site and is intended for long-term storage and for further processing, a typical example might be the output of a simulation. In addition to Fenix resources, we assume the presence of a file catalogue (or a community-specific metadata catalogue). Second, we sketch the transactions needed to achieve said goals; see also Fig. 3

1) User authenticates against his project's IdP.

2) User uploads the dataset from site $A$ to a Fenix ARD instance.

a) Authentication is forwarded to the specific ARD instance.

b) Authorisation, quota, etc is checked by the instance.

c) Access controls are set.

3) User registers the data location with the file catalogue.

4) User retrieves data location from the file catalogue, e.g. via a search.

5) User downloads the dataset from the location in the ARD to site $\mathrm{B}$.

a) Authentication is forwarded to the specific ARD instance.

b) Authorisation, quota, etc is checked by the instance.

c) Access controls are checked.

We assume here that the credentials, e.g. a token, is requested only once and used at both sites. While the index is largely superfluous in this simple example, it is crucial when the workflow is automated and interactions of the user largely replaced by scripts or if the actual data locations cannot easily be communicated between steps.

\section{Case Study: Neurorobotics}

The EBRAINS Neurorobotics Platform (NRP) is an environment for interactive in-silico experiments for studying models of brain, body, and environment interaction via closed perception-action loops [4], [8]. Scientists interact with the platform through a web-interface to control simulations of full models of robot and environment. Simulated sensors of the robot relay environment information to a simulated nervous system that models a biological brain at different levels of detail, which in turn controls the robot.

The actual workflow is sketched out in Fig. 4 and uses multiple services offered by Fenix, foremost the interactive compute, virtual machine, and scalable compute services. The user's point of interaction is a Graphical User Interface (GUI) based on web technologies, i.e. the browser, which drives the NRP frontend running in a VM. This frontend manages the setup and connections of the two coupled simulations on the HPC machines, for enviroment and neurons. Further, 


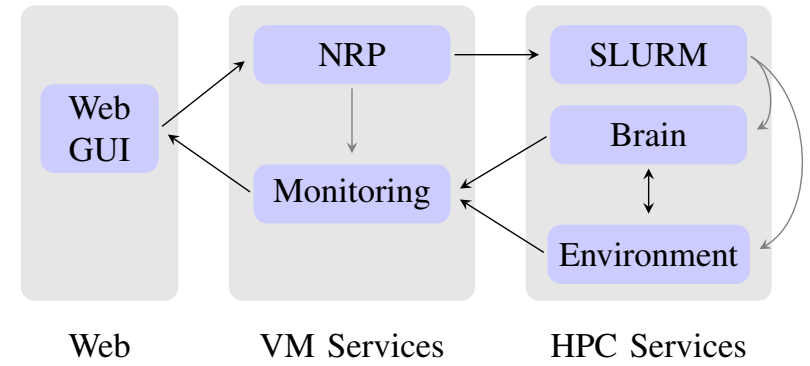

Fig. 4. Schematic of the NRP usecase workflow. A browser-based GUI is used to instantiate an experiment through the NRP frontend, which triggers the actual simulations through a workload manager (SLURM), running in the HPC environment. It will start and connect the simulation of environments and neurons. These simulations will connect to an interactive monitoring component - also prepared by the frontend - which is handed over to the user for interactive control and diagnostic of the experiment.

a connection from each of the simulation to a VM also instantiated by the frontend is made. This instance provides interactive monitoring and control to the user via the GUI.

NRP enables the following workflow for a user through a web browser:

1) The users logins into the federated IdP exploiting Fenix AAI and VM services.

2) Robot, environment, and brain models are selected for the experiment by the researcher using the Neurorobotics Cockpit (Frontend).

3) Experiment setup parameters are selected for simulation execution through the Frontend.

4) Frontend submits the experiment for execution on HPC (SCC) infrastructure.

5) Frontend configures and redirects the user to a proxy server that will become a gateway for the user to interact with the experiment being executed on the HPC infrastructure.

6) Core NRP components including brain and robot model simulations are started on HPC infrastructure (compute nodes).

7) Brain (NEST) and robot (Gazebo) simulations synchronized by the Closed Loop Engine component of NRP, where output of one simulation is used as input of the other and vice versa.

8) Researcher monitors the experiment in real-time, being able to control all states and parameters of the ongoing experiment.

\section{Vi. Case Study: Brain Atlas}

The Brain Atlas project seeks to develop a platform for interactive exploration of data resulting from high-resolution brain tissue imaging. For this, volumetric maps of the human brain must be built from actual preserved human brains.

One major component is the contiguous ingestion of new data from post-mortem microscopy of human brains into the atlas [1]. A set of microscopes at Forschungszentrum Jülich (FZJ) scans the prepared brain tissue layer by layer. As the scanning process has artefacts and distorts and shifts individual tiles, they need to be stitched into a two-dimensional image that accurately represents the original brain. A set of these images is then re-assembled into a three-dimensional map, eliminating the background using automated sgementation. This registration step forms the basis for all further steps; it is computationally intensive and usually performed on a GPUcluster. At this point the dataset is complete and usable, with a size of roughly 1 TByte per full brain section. However, further analyses are performed to augment it before presentation, e.g. extraction of cell locations and contours, annotation of cytoarchitectonic brain areas, etc. These are, again, computationally intensive processes that benefit from scalable GPU resources, i.e. machine learning, deep learning, and image processing. Orthogonal datasets, like three-dimensional fibre re-construction is treated similarly and mapped onto the volumetric representation [2]. Further, multiple full-brain maps are merged into a common reference space to reduce impact of the individual physiology.

A simplified workfow is shown in Figure 5 to explain how it is mapped to the Fenix infrastructure. Ingestion of the raw image data is performed at one site. The following analyses requires significant computational resources and can be performed in an automatised way using SCC services. To provide fast access to the data, the data mover service is used to replicate data in an ACD. Finally, all data is merged in an ARD to make the data accessible to the wider community, e.g. via viewer services exposed to the web via a GUI or as part of interactive compute jobs. Further processing for presentation in an interactive viewer is necessary, such as tiling and levelof-detail maps, for efficient retrieval and caching.

\section{RELATED WORK}

There are several other European and non-European einfrastructure projects that aim at offering a set of federated services being offered for Fenix. Organisations and projects like EGI [6], EUDAT [14], PRACE [18], XSEDE [22], JetStream cloud [11] or Nectar Cloud [15] operate different types of distributed e-infrastructure services. They all are addressing the challenge of managing federated identities and access to distributed computing and storage resources. Some of these projects do also have a research and development agenda. The number of projects that focused on research and development, like INDIGO Data Cloud [20], seems to be declining. One of the possible reasons is the growing focus on technologies that have a stronger industrial backing, like technologies developed by the OpenStack community.

Compared to the aforementioned e-infrastructure service providers, the unique aspect of Fenix architecture is a holistic view of HPC and cloud computing and storage infrastructures. While most of the existing infrastructures either focus on HPC or Cloud, Fenix aims integrating both paradigms.

\section{OUTLOOK}

A number of Fenix services are already in production and are being used by different platforms of the European HBP. Research and development projects are undergoing for 


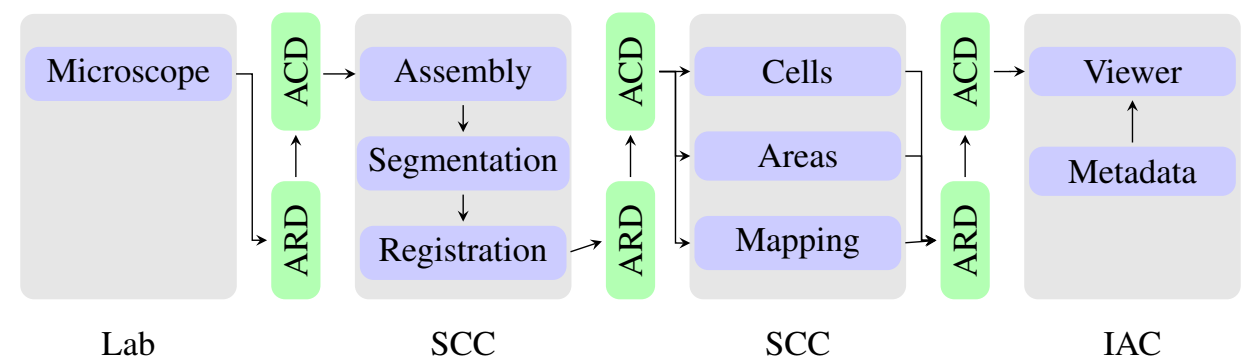

Fig. 5. Schematic of the Brain Atlas usecase workflow. In a first step, images are generated in the lab and re-assembled into a coherent dataset by an automated registration process. From here, the data is archived in the ARD and enhanced with metadata for indexing. A series of augmentations is then performed independently at multiple sites, making use of the respective SCC. All results are merged with the original data set, which is presented to researchers using a web-based renderer running on Fenix IAC. Due to the extreme size of the data presented, use of ARD is needed to hold the full data set, while the ACD is facilitate sufficiently fast access to the data.

enabling interactive computing, FURMS and Data Mover services to realise the full potential of Fenix. The next steps include alignment with the European Open Science Cloud (EOSC) [7] for offering HBP and cloud compute and storage services for an even broader set of European research communities.

\section{ACKNOWLEDGEMENTS}

Funding for the work is received from the European Commission H2020 program under Specific Grant Agreement No. 800858 (ICEI).

\section{REFERENCES}

[1] Amunts, K., Zilles, K.: Architectonic mapping of the human brain beyond brodmann. Neuron $\mathbf{8 8}$ (2015). https://doi.org/https://doi.org/10.1016/j.neuron.2015.12.001

[2] Axer, M., Strohmer, S., Gräßel, D., Bücker, O., Dohmen, M., Reckfort, J., Zilles, K., Amunts, K.: Estimating Fiber Orientation Distribution Functions in 3D-Polarized Light Imaging. Frontiers in neuroanatomy 10 (2016). https://doi.org/10.3389/fnana.2016.00040

[3] EBRAINS: https://ebrains.eu/

[4] EBRAINS: Neurorobotics Platform, https://neurorobotics.net

[5] eduTEAMS: https://eduteams.org/

[6] EGI: https://www.egi.eu/

[7] European Commission: European Open Science Cloud, https://ec.europa.eu/digital-single-market/en/ european-open-science-cloud

[8] Falotico, E., Vannucci, L., Ambrosano, A., Albanese, U., Ulbrich, S., Vasquez Tieck, J.C., Hinkel, G., Kaiser, J., Peric, I., Denninger, O., Cauli, N., Kirtay, M., Roennau, A., Klinker, G., Von Arnim, A., Guyot, L., Peppicelli, D., Martínez-Cañada, P., Ros, E., Maier, P., Weber, S., Huber, M., Plecher, D., Röhrbein, F., Deser, S., Roitberg, A., van der Smagt, P., Dillman, R., Levi, P., Laschi, C., Knoll, A.C., Gewaltig, M.O.: Connecting artificial brains to robots in a comprehensive simulation framework: The neurorobotics platform. Frontiers in Neurorobotics 11 (2017). https://doi.org/10.3389/fnbot.2017.00002

[9] Fenix: Resources, https://fenix-ri.eu/infrastructure/ resources

[10] Fenix: Virtual Machine Services, https://fenix-ri.eu/ infrastructure/services/virtual-machine-services

[11] Fischer, J., Tuecke, S., Foster, T.I., Stewart, A.C.: Jetstream: A distributed cloud infrastructure for underresourced higher education communities. SCREAM@HPDC (2015)

[12] GÉANT: https://www.geant.org/

[13] Kanellopoulos, C., Stevanovic, U., Hardt, M., et al.: AARC blueprint architectures. Tech. rep., AARC project (2017), https://aarc-project.eu/wp-content/uploads/2017/ 05/DJRA1.2-AARC-Blueprint-Architectures-1.pdf
[14] Mallmann, D., von St. Vieth, B., Riedel, M., Rybicki, J., Koski, K., Lecarpentier, D., Wittenburg, P.: EUDAT - Towards a pan-European Collaborative Data Infrastructure. Innovatives Supercomputing in Deutschland: inSiDE 10(1), 84 - 85 (2012), record converted from VDB: 12.11.2012

[15] Nectar Cloud: https://nectar.org.au/research-cloud/

[16] OpenStack: Swift, https://docs.openstack.org/swift

[17] OpenStack: Swiftonfile, https://wiki.openstack.org/wiki/ Swiftonfile

[18] PRACE: https://prace-ri.eu/

[19] REFEDS: eduPerson, https://refeds.org/eduperson

[20] Salomoni, D., Campos, I., Gaido, L., de Lucas, J.M., Solagna, P., Gomes, J., Matyska, L., Fuhrman, P., Hardt, M., Donvito, G., Dutka, L., Plociennik, M., Barbera, R., Blanquer, I., Ceccanti, A., Cetinic, E., David, M., Duma, C., López-García, A., Moltó, G., Orviz, P., Sustr, Z., Viljoen, M., Aguilar, F., Alves, L., Antonacci, M., Antonelli, L.A., Bagnasco, S., Bonvin, A.M.J.J., Bruno, R., Chen, Y., Costa, A., Davidovic, D., Ertl, B., Fargetta, M., Fiore, S., Gallozzi, S., Kurkcuoglu, Z., Lloret, L., Martins, J., Nuzzo, A., Nassisi, P., Palazzo, C., Pina, J., Sciacca, E., Spiga, D., Tangaro, M., Urbaniak, M., Vallero, S., Wegh, B., Zaccolo, V., Zambelli, F., Zok, T.: INDIGO-DataCloud: a Platform to Facilitate Seamless Access to E-Infrastructures. Journal of Grid Computing 16(3), 381-408 (sep 2018). https://doi.org/10.1007/s10723018-9453-3

[21] SATOSA: https://github.com/IdentityPython/SATOSA

[22] Stewart, C.A., Bachmann, F., Hazlewood, V., Knepper, R., Foster, I., Ferguson, J., Grimshaw, A., Lifka, D.: What is campus bridging and what is XSEDE doing about it?, p. 1. Association for Computing Machinery (ACM) (2012). https://doi.org/10.1145/2335755.2335844 